\title{
A Single Queue with Mutually Replacing $m$ Servers
}

\author{
Kailash C. Madan \\ Ahlia University, PO Box 10878, GOSI Complex, Exhibition Road \\ Manama, Kingdom of Bahrain \\ E-mail: kcmadan@yahoo.com
}

\begin{abstract}
Customers arriving in a Poisson stream are served one by one exponentially by one of the $m$ servers $S_{1}, S_{2}, S_{3}, \ldots, S_{m}$. A server who has just completed a service either continues the next service or is replaced by another server. The replacement of a server at each service completion is governed by a probabilistic criterion of availability of servers. Transient solutions in terms of Laplace transforms of the probability generating functions are obtained and among some special cases the earlier known results for the case of 2 servers are deduced. The steady state solutions and the average queue sizes have been obtained for some particular cases.
\end{abstract}

Keywords: Poisson stream, Exponential service, Availability criterion, Laplace transform, Probability generating functions, Sequential service, Steady state, Average queue size

\section{Introduction}

(Madan, 1990) has studied a 2 server queue with correlated availability of servers. The idea was motivated by some earlier papers dealing with correlated arrivals, correlated departures and some other similar situations. cf (Chaudary, 1965), (Mohan, 1955) and (Murari, 1969). For an excellent account of the single server queue, we refer the reader to (Cohen, 1969), Gross and (Harris, 1985) and (Saaty, 1961). In this paper, we generalize the problem studied by (Madan, 1990) to m servers. The mathematical model is briefly described by the following assumptions:

- Arrivals occur one by one in a Poisson stream with mean arrival rate $\lambda(>0)$.

- The system has $m$ servers designated as $S_{1}, S_{2}, S_{3}, \cdots, S_{m}$ and only one of them serves the customers at a time. The service is provided on a first come, first served basis and the service times of are exponentially distributed with mean service time $1 / \mu_{j}, j=1,2,3, \cdots, m$.

- Whenever an empty system starts with a first arriving customer, the servers $S_{1}, S_{2}, S_{3}, \cdots, S_{m}$ are available in the system with respective probabilities $\pi_{j}, j=1,2,3, \cdots, m$, where $\sum_{j=1}^{m} \pi_{j}=1$.

- However, subsequently at the completion of each service, a server who has just completed a service either continues the next service or is replaced by another server. The availability criterion of servers is determined by the conditional probability $p_{i j}=$ probability that server $S_{j}$ replaces server $S_{i}$, given that the server $S_{i}$ has just completed a service. Obviously, when $j=i$, it means that the server $S_{i}$ continues with the next service. Thus the $m \times m$ availability matrix is given by

$$
\left(\begin{array}{cccc}
p_{11} & p_{12} & \cdots & p_{1 m} \\
p_{21} & p_{22} & \cdots & p_{2 m} \\
\cdots & \cdots & \cdots & \cdots \\
p_{m 1} & p_{m 2} & \cdots & p_{m m}
\end{array}\right)
$$

- It has been assumed that the replacement of servers is instantaneous.

\section{Equations Governing the System}

We define $P_{n}^{(j)}(t), n \geq 0$ as the probability that at time t there are $n$ customers in the queue excluding the one being served by the $j$ th server $S_{j}, j=1,2,3, \cdots, m$ and let $Q(t)$ be the probability that at time $t$ the queue length is zero and none of the $m$ servers is providing service. The following set of forward equations govern the system for $j=1,2,3, \cdots, m$ :

$$
\begin{gathered}
\frac{d}{d t} P_{n}^{(j)}(t)+\left(\lambda+\mu_{j}\right) P_{n}^{(j)}(t)=\lambda P_{n-1}^{(j)}(t)+\sum_{k=1}^{m} p_{k j} \mu_{k} P_{n+1}^{(k)}(t), \quad n>0 \\
\frac{d}{d t} P_{0}^{(j)}(t)+\left(\lambda+\mu_{j}\right) P_{0}^{(j)}(t)=\lambda \pi_{j} Q(t)+\sum_{k=1}^{m} p_{k j} \mu_{k} P_{1}^{(k)}(t)
\end{gathered}
$$




$$
\frac{d}{d t} Q(t)+\lambda Q(t)=\sum_{k=1}^{m} \mu_{k} P_{0}^{(k)}(t)
$$

We assume that initially there are no customers either waiting in queue or being served so that the initial condition is

$$
Q(0)=0
$$

Taking Laplace transform of equations (1) to (3) and using equation (4), we have

$$
\begin{gathered}
\left(s+\lambda+\mu_{j}\right) P_{n}^{(j)}(s)=\lambda P_{n-1}^{(j)}(s)+\sum_{k=1}^{m} p_{k j} \mu_{k} P_{n+1}^{(k)}(S), \quad n>0 \\
\left(s+\lambda+\mu_{j}\right) P_{0}^{(j)}(s)=\lambda \pi_{j} Q(S)+\sum_{k=1}^{m} p_{k j} \mu_{k} P_{1}^{(k)}(S) \\
(s+\lambda) Q(s)=1+\sum_{k=1}^{m} \mu_{k} P_{0}^{(k)}(S)
\end{gathered}
$$

\section{The Time-Dependent Solution}

Let $\bar{P}^{(j)}(s, z)=\sum_{n=0}^{\infty} \bar{P}^{(j)}(s) z^{n},|z| \leq 1$ define the probability generating functions of queue length under various states of the system in terms of their Laplace transforms. Then multiplying equations (5) and (6) by suitable powers of $\mathrm{z}$ and simplifying, we obtain

$$
\left(s+\lambda+\mu_{j}-\lambda z\right) z P^{(j)}(s, z)=\lambda z \pi_{j} \bar{Q}(s)+\sum_{k=1}^{m} p_{k j} \mu_{k} P^{(k)}(s, z)-\sum_{k=1}^{m} p_{k j} \mu_{k} \bar{p}^{(k)}(S), \quad n>0, \quad j=1,2,3, \cdots, m
$$

We solve the system of equations given by $(8)$ for $\bar{P}^{(j)}(s, z), j=1,2, \cdots, m$ and obtain

$$
\bar{P}^{(j)}(s, z)=\frac{N^{(j)}}{\Delta}
$$

Where $\Delta$ is the determinant of the $m \times m$ matrix

$$
\begin{gathered}
{\left[\begin{array}{cccc}
G_{1} & -p_{21} \mu_{2} & \cdots & -p_{m 1} \mu_{m} \\
-p_{12} \mu_{1} & G_{2} & \cdots & -p_{m 2} \mu_{m} \\
\ldots & \cdots & \cdots & \cdots \\
-p_{1 m} \mu_{1} & -p_{2 m} \mu_{2} & \cdots & G_{m}
\end{array}\right]} \\
G_{j}=\left(s+\lambda+\mu_{j}-\lambda z\right) z-p_{j j} \mu_{j} \\
H_{j}=\lambda z \pi_{j} \bar{Q}(s)-\sum_{k=1}^{m} p_{k j} \mu_{k} \bar{P}_{0}^{(k)}(S)
\end{gathered}
$$

and $N^{(j)}$ is the $m \times m$ determinant which is obtained from the determinant $\Delta$ by replacing the $j$ th column of $\Delta$ by the column vector $\left(H_{1}, H_{2}, \cdots, H_{m}\right)^{\prime}$.

We note that due to the vector $\left(H_{1}, H_{2}, \cdots, H_{m}\right)^{\prime}$ appearing in $N^{(j)}$, the numerator of each of the equations given by (9) involves $m+1$ unknowns, namely $\bar{Q}(s)$, and $\bar{P}_{0}^{(k)}(s), k=1,2, \cdots, m$. We proceed to determine these unknowns. It is easy to see that each $G_{j}=\left(s+\lambda+\mu_{j}-\lambda z\right)-p_{j j} \mu_{j}$ has only one zero inside the unit circle $|z|=1$ for $j=1,2,3, \cdots, m$ and, for that matter, due to the product term $G_{1} G_{2} \cdots G_{m}$ appearing in $\Delta$, the denominator of the right side of (9) has $m$ zeros inside $|z|=1$. These zeros give rise to $m$ equations in $m+1$ unknowns mentioned above. In addition, equation (6) 
also involves the same $m+1$ unknowns. Thus there are in all $m+1$ equations in $m+1$ unknowns. Hence all $m$ probability generating functions $\bar{P}^{(j)}(s, z), j=1,2, \cdots, m$ can be completely determined.

\section{Some Particular Cases}

\subsection{Case 1: (Sequential Service)}

If we let $p_{12}=p_{23}=p_{34}=\cdots=p_{m-1, m}=1$ and all other transition probabilities are set to zero, then this essentially means that the servers are providing sequential service, one after the other. In this case, the corresponding results will be given by (9) where, now will have

$$
\begin{gathered}
G_{j}=\left(s+\lambda+\mu_{j}-\lambda z\right) z, \quad j=1,2, \cdots, m, \\
H_{j}=\lambda z \pi_{j} \bar{Q}(s)-\mu_{j-1} \bar{P}_{0}^{(j-1)}(s), \quad j=2,3,4, \cdots, m, \\
H_{1}=\lambda z \pi_{1} \bar{Q}(s)-\mu_{m} \bar{P}_{0}^{(m)}(s) .
\end{gathered}
$$

\subsection{Case 2: (each server completes his cycle)}

Let $p_{i i}=1$ for $i=2,3,4, \cdots, m$ and $p_{i j}=0$ for $i \neq j$ which means that $S_{1}, S_{2}, S_{3}, \cdots, S_{m}$, whosoever starts service continues serving the customers until the queue becomes empty again. In this case,

$$
\begin{gathered}
G_{j}=\left(s+\lambda+\mu_{j}-\lambda z\right) z-\mu_{j} \\
H_{j}=\lambda z \pi_{j} \bar{Q}(s)-\mu_{j} \bar{P}_{0}^{(j)}(s), \quad j=1,2,3,4, \cdots, m .
\end{gathered}
$$

And then (9) yields

$$
\bar{P}^{(j)}(s, z)=\frac{\lambda z \pi_{j} \bar{Q}(s)-\mu_{j} \bar{P}_{0}^{(j)}(s)}{\left(s+\lambda+\mu_{j}-\lambda z\right)-\mu_{j}}
$$

The denominator of the right side of equation (10) has one zero inside the unit circle $|z|=1$. Let $z=z_{j}$ be this zero. This zero yields

$$
\lambda z \pi_{j} \bar{Q}(s)-\mu_{j} \bar{P}_{0}^{(j)}(s)=0 \text { for } j=1,2, \cdots, m
$$

Using equation (11) in (7), we obtain

$$
\bar{Q}(s)=\frac{1}{s+\lambda-\lambda \sum_{j=1}^{m} \pi_{j} z_{j}}
$$

And hence

$$
\bar{P}_{0}^{(j)}(s)=\frac{\lambda z_{j} \pi_{j}}{s+\lambda-\lambda \sum_{j=1}^{m} \pi_{j} z_{j}}, j=1,2, \cdots, m
$$

\subsubsection{Steady State Queue Length Probabilities for Various States of the System}

Let $P_{n}^{(j)}, j=1,2, \cdots, m$ and $Q$ be the respective steady state probabilities corresponding to $P_{n}^{(j)}(t)$ and $Q(t)$ and, for that matter, let $P^{(j)}(z)$ be the steady state probability generating functions for the queue length corresponding to $P^{(j)}(s, z)$. Then the steady state solution can be obtained by using the well known Tauberian property $\lim _{s \rightarrow \infty} s f(s)=\lim _{t \rightarrow \infty} f(t)$.

We proceed to derive the steady state results for this case as follows: Applying the Tauberian property, equation (10) yields

$$
P^{(j)}(z)=\frac{\lambda z \pi_{j} Q-\mu_{j} P_{0}^{(j)}}{\left(\lambda+\mu_{j}-\lambda z\right)-\mu_{j}}, \quad j=1,2, \cdots, m
$$

Now, $z=1$ is a zero of the denominator of the right hand side of equation (14). Therefore, its denominator must vanish for this zero, giving

$$
P_{0}^{(j)}=\left(\frac{\lambda}{\mu_{j}}\right) \pi_{j} Q, \quad j=1,2, \cdots, m
$$


Using (15), equation (14) can be written as

$$
P^{(j)}(z)=\frac{\lambda(z-1) \pi_{j} Q}{\left(\lambda+\mu_{j}-\lambda z\right)-\mu_{j}}, \quad j=1,2, \cdots, m
$$

We see than for $z=1$, equation (16) is indeterminate of the $0 / 0$ form. Therefore, using L'Hopital's rule, we obtain

$$
P^{(j)}(1)=\frac{\lambda \pi_{j} Q}{\mu_{j}-\lambda}, \quad j=1,2, \cdots, m
$$

Next, utilizing (17) in the normalizing condition $\sum_{j=1}^{m} P^{(j)}(1)+Q=1$, we obtain

$$
Q=\frac{1}{1+\lambda \sum_{j=1}^{m}\left(\frac{\pi_{j}}{\mu_{j}-\lambda}\right)}
$$

We note that equation (18) gives the steady state probability that the system is empty and none of the servers is providing service.

Then using the value of $Q$ from (18) in equation (16), we get

$$
P^{(j)}(z)=\left[\frac{\lambda(z-1) \pi_{j}}{\left(\lambda+\mu_{j}-\lambda z\right)-\mu_{j}}\right]\left[1+\lambda \sum_{j=1}^{m}\left(\frac{\pi_{j}}{\mu_{j}-\lambda}\right)\right]^{-1}, \quad j=1,2, \cdots, m
$$

Factoring $\left(\lambda+\mu_{j}-\lambda z\right) z-\mu_{j}$ as $(z-1)\left(\mu_{j}-\lambda z\right)$ and canceling out the factor $(z-1)$, we can re-write equation (19) as

$$
P^{(j)}(z)=\left[\frac{\lambda \pi_{j}}{\left(\mu_{j}-\lambda z\right)}\right]\left[1+\lambda \sum_{j=1}^{m}\left(\frac{\pi_{j}}{\mu_{j}-\lambda}\right)\right]^{-1}, \quad j=1,2, \cdots, m
$$

This can be again written as

$$
P^{(j)}(z)=\left[\frac{\lambda \pi_{j}}{\mu_{j}}\right]\left[1+\lambda \sum_{j=1}^{m}\left(\frac{\pi_{j}}{\mu_{j}-\lambda}\right)\right]^{-1}\left(1-\frac{\lambda z}{\mu_{j}}\right)^{-1}, n \geq 0, j=1,2, \cdots, m
$$

Expanding the last factor of the right hand side of equation (21) and picking up the coefficients of nth power of $\mathrm{z}$ and simplifying, we have

$$
P_{n}^{(j)}=\pi_{j}\left[1+\lambda \sum_{j=1}^{m}\left(\frac{\pi_{j}}{\mu_{j}-\lambda}\right)\right]^{-1}\left(\frac{\lambda}{\mu_{j}}\right)^{n+1}, n \geq 0, j=1,2, \cdots, m
$$

\subsubsection{Steady State Mean Queue Length}

Let $p_{n}=\sum_{j=1}^{n} p_{n}^{(j)}$ be the steady state probability that the queue length is $n \geq 0$, irrespective of whosoever server is providing service. Then the mean queue lengh $L_{q}$ is given by

$$
L_{q}=\sum_{n=0}^{\infty} n p_{n}=\sum_{(n=0)}^{\infty} \sum_{j=1}^{m} n \pi_{j}\left[1+\lambda \sum_{(j=1)}^{m}\left(\frac{\pi_{j}}{\mu_{j}-\lambda}\right)\right]^{-1}\left(\frac{\lambda}{\mu_{j}}\right)^{n+1}
$$

Carrying out the summations and simplifying, we have the mean queue length as

$$
L_{q}=\left[1+\lambda \sum_{(j=1)}^{m}\left(\frac{\pi_{j}}{\mu_{j}-\lambda}\right)\right]^{-1} \sum_{j=1}^{m} \pi_{j}\left(\frac{\lambda}{\mu_{j}-\lambda}\right)^{2}
$$




\subsection{Case 3: (The Case of Two Servers)}

If the system has two servers, then $m=2$ and, for that matter, $\pi_{j}=0=P^{(j)}(s, z)$, for $j=3, \cdots, m$. Also $p_{i j}=0$ for $i, j=3, \cdots, m$ so that now we have $2 \times 2$ selection matrix given by $\left(\begin{array}{ll}p_{11} & p_{12} \\ p_{21} & p_{22}\end{array}\right)$. Then in this case, the equations in matrix form will be

$$
\left(\begin{array}{cc}
G_{1} & -p_{21} \mu_{2} \\
-p_{12} \mu_{1} & G_{2}
\end{array}\right)\left(\begin{array}{l}
\bar{P}^{(1)}(s, z) \\
\bar{P}^{(2)}(s, z)
\end{array}\right)=\left(\begin{array}{l}
H_{1} \\
H_{2}
\end{array}\right)
$$

Where

$$
\begin{gathered}
G_{1}=\left(s+\lambda+\mu_{1}-\lambda z\right) z-p_{11} \mu_{1} \\
G_{2}=\left(s+\lambda+\mu_{2}-\lambda z\right) z-p_{22} \mu_{2} \\
H_{1}=\lambda z \pi_{1} \bar{Q}(s)-p_{11} \mu_{1} \bar{P}_{0}^{(1)}(s)-p_{21} \mu_{2} \bar{P}_{0}^{(2)}(s) \\
H_{2}=\lambda z \pi_{2} \bar{Q}(s)-p_{12} \mu_{1} \bar{P}_{0}^{(1)}(s)-p_{22} \mu_{2} \bar{P}_{0}^{(2)}(s)
\end{gathered}
$$

Solving (25) simultaneously, we have

$$
\begin{aligned}
& \bar{P}^{1}(s, z)=\frac{H_{1}\left[\left(s+\lambda+\mu_{2}-\lambda z\right) z-p_{22} \mu_{2}\right]+p_{21} \mu_{2} H_{2}}{\left[\left(s+\lambda+\mu_{1}-\lambda z\right) z-p_{11} \mu_{1}\right]\left[\left(s+\lambda+\mu_{2}-\lambda z\right) z-p_{22} \mu_{2}\right]} \\
& \bar{P}^{2}(s, z)=\frac{H_{2}\left[\left(s+\lambda+\mu_{1}-\lambda z\right) z-p_{11} \mu_{1}\right]+p_{12} \mu_{1} H_{1}}{\left[\left(s+\lambda+\mu_{1}-\lambda z\right) z-p_{11} \mu_{1}\right]\left[\left(s+\lambda+\mu_{2}-\lambda z\right) z-p_{22} \mu_{2}\right]}
\end{aligned}
$$

Results in (26) and (27) agree with known results of (Madan, 1990)

Under the conditions of particular case 2 (each server completes his cycle), we have $p_{11}=1=p_{22}$ and $p_{12}=0=p_{21}$, for $j=2$. Consequently, the steady state results corresponding to equations (16), (18), (22) and (24) can be derived as follows:

$$
\begin{gathered}
P^{(j)}(z)=\frac{\lambda(z-1) \pi_{j} Q}{\left(\lambda+\mu_{j}-\lambda z\right)-\mu_{j}}, \quad j=1,2 \\
Q=\frac{\left(\mu_{1}-\lambda\right)\left(\mu_{2}-\lambda\right)}{\lambda \pi_{1}\left(\mu_{2}-\lambda\right)+\lambda \pi_{2}\left(\mu_{1}-\lambda\right)+\left(\mu_{1}-\lambda\right)\left(\mu_{2}-\lambda\right)} \\
p_{n}^{(j)}(z)=\pi_{j}\left[\frac{\left(\mu_{1}-\lambda\right)\left(\mu_{2}-\lambda\right)}{\lambda \pi_{1}\left(\mu_{2}-\lambda\right)+\lambda \pi_{2}\left(\mu_{1}-\lambda\right)+\left(\mu_{1}-\lambda\right)\left(\mu_{2}-\lambda\right)}\right]\left(\frac{\lambda}{\mu_{j}}\right)^{n+1}, n \geq 0, \quad j=1,2 \\
L_{q}=\left[\frac{\left(\mu_{1}-\lambda\right)\left(\mu_{2}-\lambda\right)}{\lambda \pi_{1}\left(\mu_{2}-\lambda\right)+\lambda \pi_{2}\left(\mu_{1}-\lambda\right)+\left(\mu_{1}-\lambda\right)\left(\mu_{2}-\lambda\right)}\right]\left[\sum_{j=1}^{2} \pi_{j}\left(\frac{\lambda}{\mu_{j}-\lambda}\right)^{2}\right]
\end{gathered}
$$

Again, all the above results given in equations (28), (29), (30) and (31) agree with the results of (Madan, 1990) except for notations.

\section{References}

Chaudhary, M. L. (1965). Correlated Queueing. Journal of the Canadian Operations Research Society, 3, No. 2. pp. 11-18.

Cohen, J. W. (1969). The Single Server Queue. North-Holland Publications, Amsterdam.

Gross, D. and Harris, C. M. (1985). The Fundamentals for Queueing Theory, second edition, John Wiley and Sons, New York.

Madan, K. C. (1990). A Two Server Queue with Correlated Availability of Servers. J. Indian Society of Statistics and Operations Research, XI, Nos. 1-4, 11-19. 
Mohan C. (1955). The Gambler's Ruin Problem with Correlation, Biometrica, 42, Parts 3 and 4.

Murari K. (1969). A Queueing Problem with Correlated Arrivals and General Service Time Distribution, ZAMM, 49, Heft 3, 151-156.

Saaty, T. L. (1961). Elements of Queueing Theory, Mc-Graw Hill Book Company, New York. 